\title{
A Predictive Model for the Compressive Strength of 3D Woven Textile Composites
}

\author{
Wooseok P. $\mathrm{Ji}^{1}$ and Anthony M. Waas ${ }^{2}$ \\ University of Michigan, Ann Arbor, MI 48109
}

\begin{abstract}
The compressive response of 3D woven textile composites (3DWTC), that consist of glass fiber tows and an epoxy matrix material, is studied using a finite element (FE) based micromechanics model. A parametric Representative Unit Cell (RUC) model is developed in a fully three-dimensional setting with geometry and textile architecture for modeling the textile microstructure. The RUC model also accoutns for the nonlinear behavior of the fiber tows and matrix. The computational model is utilized to predict the compressive strength of 3DWTC and its dependence on various geometrical and material parameters. The finite element model is coupled with a probabilistic analysis tool to provide probabilistic estimates for 3DWTC compressive strength.
\end{abstract}

\section{Introduction}

$\mathrm{T}$ hree-dimensional woven textile composites (3DWTCs) are gaining ever-increasing attention from various engineering sectors due to numerous structural advantages of the material system over conventional laminated composites ${ }^{1-3}$. Typical laminated composites have very good in-plane properties in the direction of the fiber tows, but tend to have low strength properties perpendicular to the fiber direction. The interfaces between the layers are the weakest link and delamnation is a common failure mode of laminated composites. Textile composites can have fiber tows in multiple directions and thus can have better properties in all loading directions. Through 3D weaving processes, 3DWTCs can implement Z-yarns woven around warp and weft fiber tows, improving the resistance to delamination failure dramatically. Advanced weaving technologies make the tow architecture tailorable to achieve desired mechanical performance for numerous applications. However, it is not easy to design and tailor 3DWTC for specific applications since it is very difficult to predict the mechanical performance of textile composites due to the complex textile architecture. Currently textile composites, especially with three dimensional architectures, are designed by an expensive build-and-test approach, which is a time consuming and costly process. There exists a stong need for a predictive model that is capable of reliably predicting mechanical performance of textile composites including basic stiffness and strength properties.

In the present study, a finite element model for 3DWTCs is developed to predict the mechanical properties, specifically with a focus on compressive strength prediction. The modeling strategy, originally developed by Song et. al. ${ }^{1}$ for two-dimensional tri-axially braied textile composites, will be extended here for 3DWTCs. They have shown good correlations with 2D in-plane woven systems for determining stiffness and strength properties, and also in describing progressive damage. The proposed model utilizes the true measured 3D geometry and nonlinear behavior of individual constituents to estimate compressive strength.

\section{Characterization and Modeling of 3\% Z-yarn 3D Woven Textile Composite}

In this paper, the orthogonally woven 3\% Z-yarn textile composite is chosen to demonstrate the modeling methodology for characterizing and predicting the mechanical properties of 3D textile composites. The selected textile composite is composed of S-2 glass fiber tows with SC-15 epoxy resin. Figure 1 shows the fiber tow architecture of the 3\% 3DWTC. The textile composite has five warp stuffers and six weft tows. A single Z-yarn is woven around each column of the weft tows. The Z-yarn has a curved path line, not simply vertically straight through the thickness of the panel. Two additional Z-yarns weave the top and bottom weft tows. The woven textile composite has $3 \%$ of $Z$-yarn fraction relative to the total fiber volume.

\footnotetext{
${ }^{1}$ Research Fellow, Department of Aerospace Engineering, 2040 FXB.

${ }^{2}$ Felix Pawlowski Collegiate Professor, Department of Aerospace Engineering, 3044 FXB, Fellow of AIAA.
} 


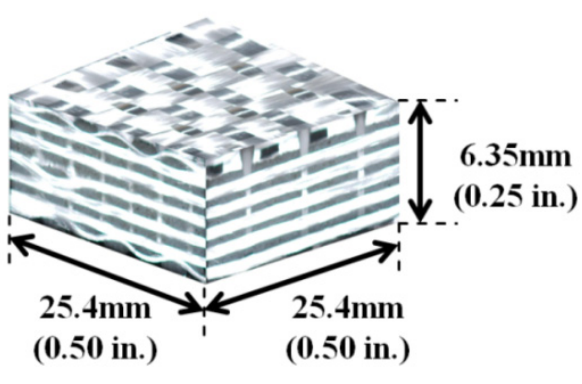

(a)

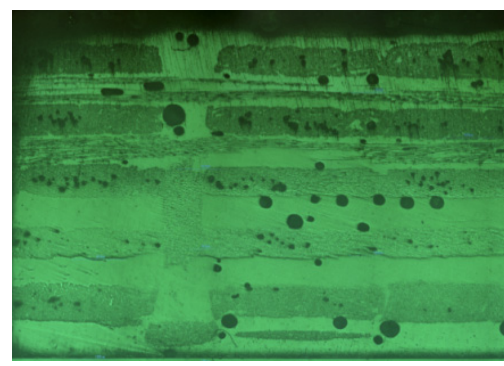

(b)

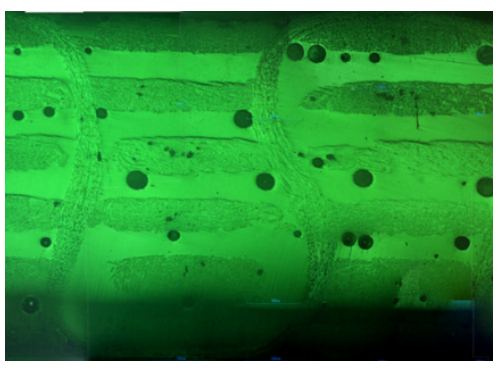

(c)

Figure 1. (a) Orthogonal woven 3\% Z-yarn textile composite. The pictures are taken after burning out the matrix material of a cured specimen. ${ }^{5}$ (b) Cross section view cut perpendicular to the warp tow direction. (c) Cross section cut perpendicular to the weft tow direction.

\section{A. Geometrical Data of Fiber Tow Architecture}

Since the 3DWTC has a nearly periodic architecture as shown in Figure 1, a finite element based representative unit cell (RUC) model is developed here to examine the mechanical response of the textile composite under compression. Detailed finite element models to represent the complex architecture of the 3DWTC require comprehensive geometrical measurements of actual specimens from microscopic images of cross sections at different locations. Thus, specimens are cut into multiple sections as shown in Figure 2 and Figure 3 and then these sections are observed under an optical microscope to extract microstructural geometric details. The geometrical measurements are listed in Table 1, Table 2 and Table 3.

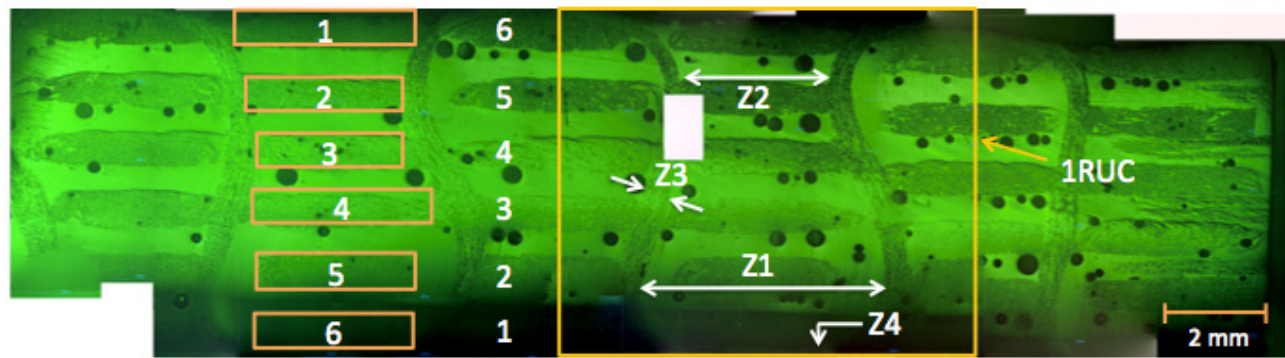

Figure 2. Cross section view of the 3DWTC perpendicular to the weft tow direction.

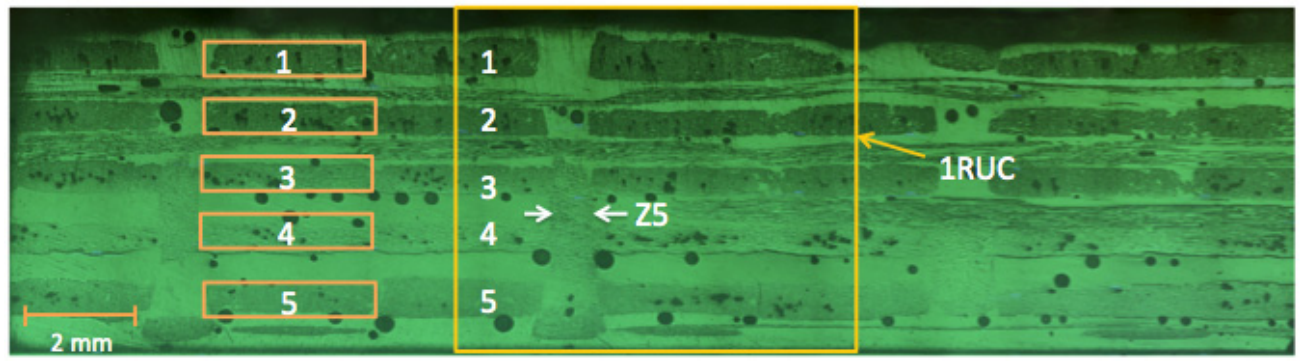

Figure 3. Cross section view of the 3DWTC perpendicular to the warp tow direction.

Table 1. Widths and heights of weft fiber tows shown in Figure 2. The tow number 1 refers to the weft tow that is not wound up by the $Z$-yarn and the tow number 6 is in direct contact with the $Z$-yarn on the surface.

\begin{tabular}{c|c|c}
\hline \hline Tow \# & Width & Height \\
\hline 1 & $3.22 \pm 0.32$ & $0.63 \pm 0.02$ \\
\hline 2 & $2.71 \pm 0.22$ & $0.67 \pm 0.04$ \\
\hline 3 & $2.89 \pm 0.21$ & $0.67 \pm 0.02$ \\
\hline 4 & $3.46 \pm 0.24$ & $0.63 \pm 0.03$ \\
\hline 5 & $3.31 \pm 0.27$ & $0.70 \pm 0.05$ \\
\hline 6 & $3.35 \pm 0.21$ & $0.68 \pm 0.04$ \\
\hline \hline \multicolumn{3}{|c}{2}
\end{tabular}

American Institute of Aeronautics and Astronautics 
Table 2. Geometrical data of warp tows shown in Figure 3. Spacing is measured for the distances between the tows separated by the Z-yarns.

\begin{tabular}{c|c|c|c}
\hline \hline Tow \# & Width & Height & Space \\
\hline 1 & $3.12 \pm 0.04$ & $0.68 \pm 0.10$ & $0.96 \pm 0.01$ \\
\hline 2 & $3.31 \pm 0.04$ & $0.55 \pm 0.02$ & $0.85 \pm 0.08$ \\
\hline 3 & $3.35 \pm 0.06$ & $0.55 \pm 0.03$ & $0.80 \pm 0.08$ \\
\hline 4 & $3.33 \pm 0.07$ & $0.56 \pm 0.02$ & $0.88 \pm 0.13$ \\
\hline 5 & $3.20 \pm 0.05$ & $0.65 \pm 0.07$ & $1.08 \pm 0.12$ \\
\hline \hline
\end{tabular}

Table 3. Geometrical characteristics of the Z-yarns. The dimensions are marked in Figure 2 and Figure 3.

\begin{tabular}{c|c|c}
\hline \hline Item \# & Dimension & Remark \\
\hline Z1 & $4.53 \pm 0.05$ & Max. gap \\
\hline Z2 & $2.86 \pm 0.11$ & Min. gap \\
\hline Z3 & $0.46 \pm 0.08$ & Avg. thickness of vertical path \\
\hline Z4 & $0.18 \pm 0.02$ & Avg. thickness of horizontal path \\
\hline Z5 & $0.91 \pm 0.14$ & Width \\
\hline \hline
\end{tabular}

\section{B. In-situ Matrix Properties}

It is well known that the in-situ response of a polymber resin in a fiber reinforced composite has effective properties that are different from those of the virgin resin material. ${ }^{1}$ This property change results from numerous reasons such as curing process-induced residual stresses, unwanted randomly distributed micro-voids, rearrangement of the polymeric chain structure with the presence of fiber fillaments and so on. The shear stressstrain responses of the virgin and in-situ SC-15 matrix are compared in Figure 4. It is easily expected that the compressive strength of the textile composite would be overpredicted if the virgin matrix properties are used. Therefore, the in-situ properties are crucial for accurately predicting compressive strength of the textile composite. In this presentation, the in-situ matrix properties of the SC-15 based on the data in Figure 4 are used throughout the RUC simulations, where the matrix is modeled as an isotropic solid with a nonlinear response in shear. For convenience, a small strain elastic-plastic characterization, that uses $J 2$ incremental theory of plasticity with a von Mises yield criterion and isotropic hardening, is adopted. The elastic properties of the isotropic in-situ matrix are obtained from the initial proportional region of the curve and they are listed in Table 4.

Table 4. Elastic properties of the SC-15 epoxy resin.

\begin{tabular}{c|c}
\hline \hline Young's modulus $(\mathrm{GPa})$ & Poisson's ratio \\
\hline 2.49 & 0.35 \\
\hline \hline
\end{tabular}

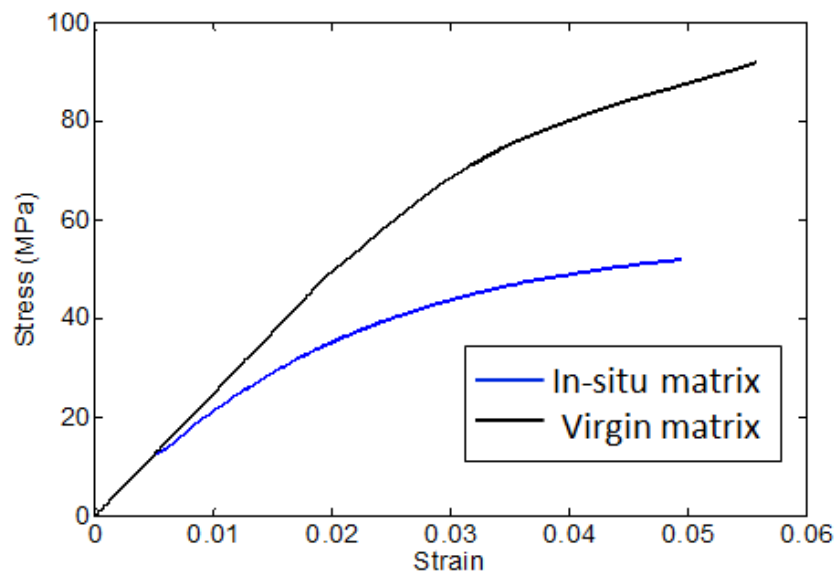

Figure 4. Nonlinear behavior of virgin and in-situ SC-15 epoxy resin., 


\section{Transversely Isotropic Fiber Tows with Nonlinear Behavior}

The warp and weft tows are composed of glass fibers with the $56 \%$ fiber volume fraction and matrix binding them altogether as shown in Figure 5. The glass fiber can be assumed as a linear elastic isotropic material and the properties are listed in Table 5. It would be practically impossible to model the large number of micro-fibers individually for a FE model in the meso-scale level. Instead of accounting for the individual fibers, the present modeling methodology treats the fiber tows as one homogenized entity with the assumption of transverse isotropy. Since the epoxy resin displays a nonlinear response as shown in Figure 4, the fiber tows, combination of fibers and matrix, also exhibit nonlinear behavior. The directional nonlinear response of the fiber tows is characterized by Hill's anisotropic plasticity model, specializing it to the case of transverse isotropy.

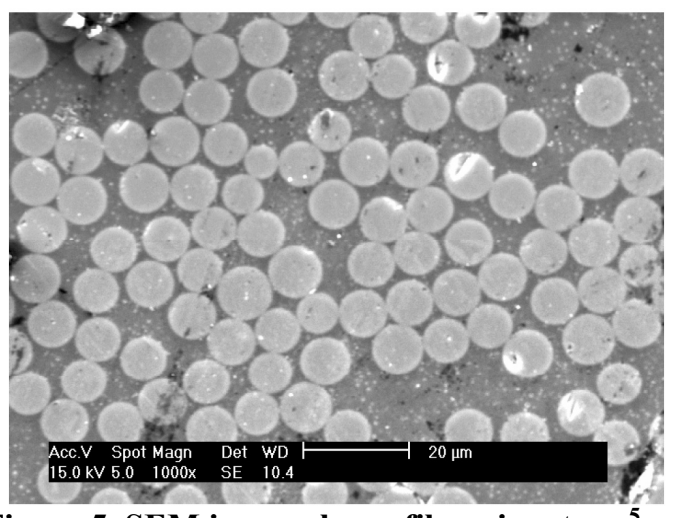

Figure 5. SEM image shows fibers in a tow. ${ }^{5}$

Table 5. Elastic properties of a S-2 glass fiber.

\begin{tabular}{c|c}
\hline \hline Young's modulus (GPa) & Poisson's ratio \\
\hline 114.2 & 0.22 \\
\hline \hline
\end{tabular}

Hill's anisotropic plasticity theory describes the yielding and plastic flow of an anisotropic material on a macroscopic scale. In the theory, a yield criterion is postulated on general grounds which is similar in form to the von-Mises criterion for isotropic materials, but which contains six parameters specifying the state of plastic anisotropy. Hill's potential function for an anisotropic material is expressed as,

$$
f(\boldsymbol{\sigma})=\sqrt{F\left(\sigma_{22}-\sigma_{33}\right)^{2}+G\left(\sigma_{33}-\sigma_{11}\right)^{2}+H\left(\sigma_{11}-\sigma_{22}\right)^{2}+2 L \sigma_{23}^{2}+2 M \sigma_{31}^{2}+2 N \sigma_{12}^{2}}
$$

where $F, G, H, L, M$, and $N$ are the six parameters obtained by tests of the material in different orientations. $F, G, H, L, M$, and $N$ can be considered as relative ease/difficulty of yielding in different directions. They are defined as

$$
\begin{gathered}
F=\frac{\left(\sigma^{0}\right)^{2}}{2}\left(\frac{1}{\bar{\sigma}_{22}^{2}}+\frac{1}{\bar{\sigma}_{33}^{2}}-\frac{1}{\bar{\sigma}_{11}^{2}}\right)=\frac{1}{2}\left(\frac{1}{R_{22}^{2}}+\frac{1}{R_{33}^{2}}-\frac{1}{R_{11}^{2}}\right) \\
G=\frac{\left(\sigma^{0}\right)^{2}}{2}\left(\frac{1}{\bar{\sigma}_{33}^{2}}+\frac{1}{\bar{\sigma}_{11}^{2}}-\frac{1}{\bar{\sigma}_{22}^{2}}\right)=\frac{1}{2}\left(\frac{1}{R_{33}^{2}}+\frac{1}{R_{11}^{2}}-\frac{1}{R_{22}^{2}}\right) \\
H=\frac{\left(\sigma^{0}\right)^{2}}{2}\left(\frac{1}{\bar{\sigma}_{11}^{2}}+\frac{1}{\bar{\sigma}_{22}^{2}}-\frac{1}{\bar{\sigma}_{33}^{2}}\right)=\frac{1}{2}\left(\frac{1}{R_{11}^{2}}+\frac{1}{R_{22}^{2}}-\frac{1}{R_{33}^{2}}\right) \\
L=\frac{3}{2}\left(\frac{\tau^{0}}{\bar{\sigma}_{23}}\right)^{2}=\frac{3}{2 R_{23}^{2}} \\
M=\frac{3}{2}\left(\frac{\tau^{0}}{\bar{\sigma}_{13}}\right)^{2}=\frac{3}{2 R_{13}^{2}}
\end{gathered}
$$




$$
N=\frac{3}{2}\left(\frac{\tau^{0}}{\bar{\sigma}_{12}}\right)^{2}=\frac{3}{2 R_{12}^{2}}
$$

where $\bar{\sigma}_{i j}$ is the measured yield stress value when $\sigma_{i j}$ is applied as the only nonzero stress component; $\sigma^{0}$ is the user-defined reference yield stress; $R_{i j}$ is the anisotropic yield stress ratio; and $\tau^{0}=\sigma^{0} / \sqrt{3}$. The six yield stress ratios are, therefore, defined as follows:

$$
\frac{\bar{\sigma}_{11}}{\sigma_{0}}, \frac{\bar{\sigma}_{22}}{\sigma_{0}}, \frac{\bar{\sigma}_{33}}{\sigma_{0}}, \frac{\bar{\sigma}_{12}}{\tau_{0}}, \frac{\bar{\sigma}_{13}}{\tau_{0}}, \frac{\bar{\sigma}_{23}}{\tau_{0}}
$$

In order to obtain the constants of Hill's potential, we need stress-strain curves of the tows in three directions, which will provide the corresponding yield strengths. Virtual tests are performed to obtain the stress-strain responses in the different directions of transverse isotropy of the fiber tows. Figure 6 shows the fiber/matrix level micromechanics model for the virtual tests. $\bar{\sigma}_{i j}$ values are obtained from each stress-strain curve where $\sigma_{i j}$ is the only nonzero stress component applied on the micromechanics model. Tension in 11 and 22 directions and shear tests in 12 and 23 directions need to be performed to fully characterize transversely isotropic nonlinear behavior of the fiber tows. Once the stress-strain data are obtained, the elastic properties of the transversely isotropic fiber tows can also be determined from the initial linear region of the curves. Figure 7 shows the results of the virtual tests for the fiber tows. The yielding points for each direction are marked with circles for each stress-strain curve. Table 6 and Table 7 summarize the result of the material characterization of the fiber tow.

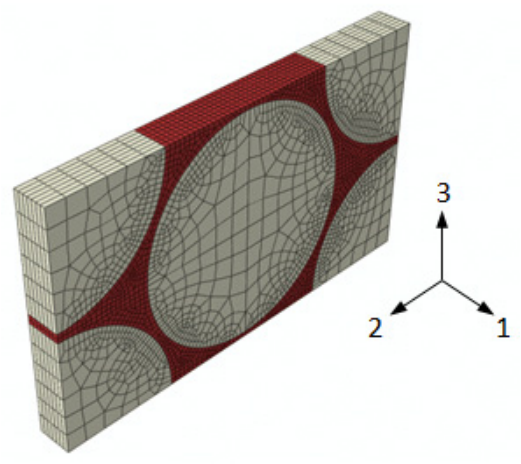

Figure 6. Fintie element fiber/matrix micromechanics model.

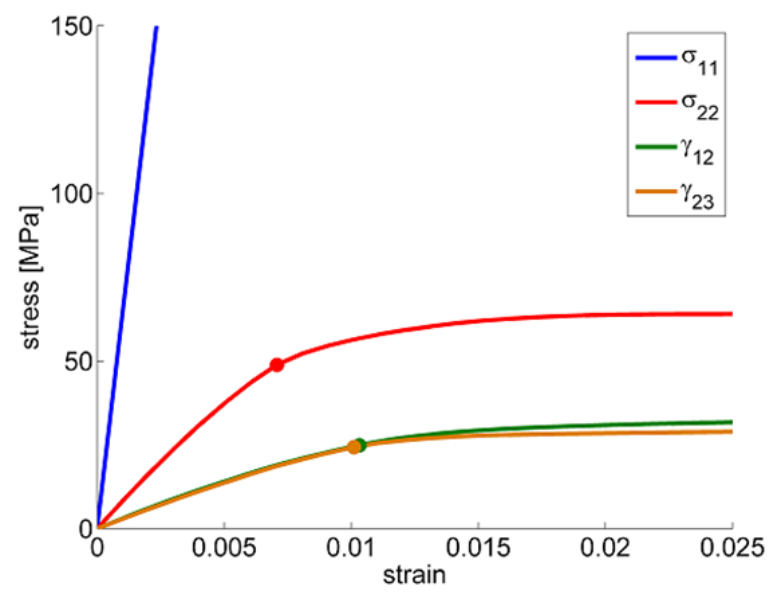

Figure 7. Results of virtual experiments on the micromechanics model.

Table 6. Transversely isotropic properties of the S-2 glass fiber.

\begin{tabular}{c|c|c|c|c}
\hline \hline $\mathrm{E}_{1}(\mathrm{GPa})$ & $\mathrm{E}_{2}(\mathrm{GPa})$ & $\mathrm{v}_{12}$ & $\mathrm{G}_{12}(\mathrm{GPa})$ & $\mathrm{G}_{23}(\mathrm{GPa})$ \\
\hline 64.4 & 8.27 & 0.27 & 3.03 & 3.03 \\
\hline \hline
\end{tabular}


Table 7. Constants for Hill's plastic potential.

\begin{tabular}{c|c|c|c|c|c}
\hline \hline R11 & R22 & R33 & R12 & R13 & R23 \\
\hline 10000 & 1.0 & 1.0 & 0.882 & 0.882 & 0.862 \\
\hline \hline
\end{tabular}

\section{Finite Element-based RUC Model}

Figure 8 shows the FE RUC model that is comprised of linear tetrahedral elements. The mesh size is sufficiently fine such that finer meshes make no more significant changes in end results. It is to be noted that the RUC model is fully parameterized with the material and geometrical data as inputs. With these input data, the construction procedure of the RUC FE model is fully automated using a python script within ABAQUS/CAE software. This feature is essential in performing a probabilistic finite element analysis where the geometries of the tows are considered as random variables, and thus the geometries of the FE model needs to be altered as necessary.

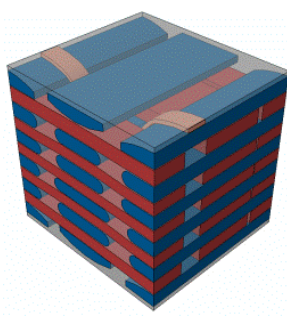

(a)
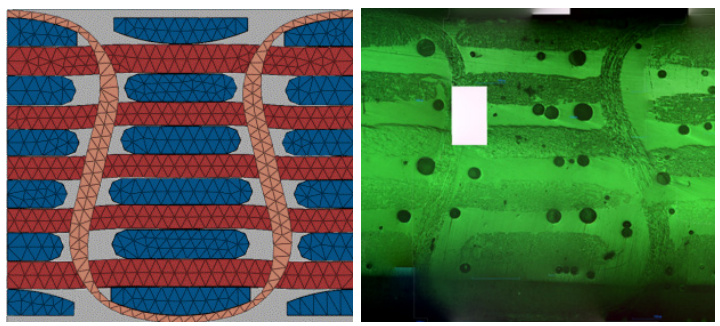

(b)

Figure 8. Finite element based RUC model. (a) Isometric view (b) The path of Z-yarn is interpolated by accounting for the different sizes and locations of the weft tows as observed in the microscopic images of actual specimens.

\section{Compressive Response of the 3DWTC}

\section{A. Quasi-static Compression Tests}

Quasi-static compression tests were carried out at a rate of $0.0004 \mathrm{in} / \mathrm{sec}$ on a hydraulically driven load frame. Specimens measured 6 inch by 4 inch and 0.235 inch thick. Specimens were placed in a compression fixture with anti-buckling guides to prevent premature failure. Curved notches were introduced to the specimens as shown in Figure 9 to promote failure in the center region of the panels. Figure 10 shows the macroscopic compressive stressstrain response of the specimens when the loading directions are aligned with the directions of the warp and weft tows. Here, the macroscopic stress is the applied load divided by the cross-sectional area of the specimens while the macroscopic strain is the normalized (by the specimen length) end-shortening. The failure mechanism was kink band formation in the axial tows (which are aligned with the load axis) that can be clearly seen in Figure 9 and Figure 10. As shown in Figure 10, the weft specimen produced a larger failure load, due to the larger volume fraction of the fiber tows in that direction. Figure 11 shows the real-time strain field of the weft specimen, where the high strain concentration is observed near the peak load, leading to the kink band failure afterwards.
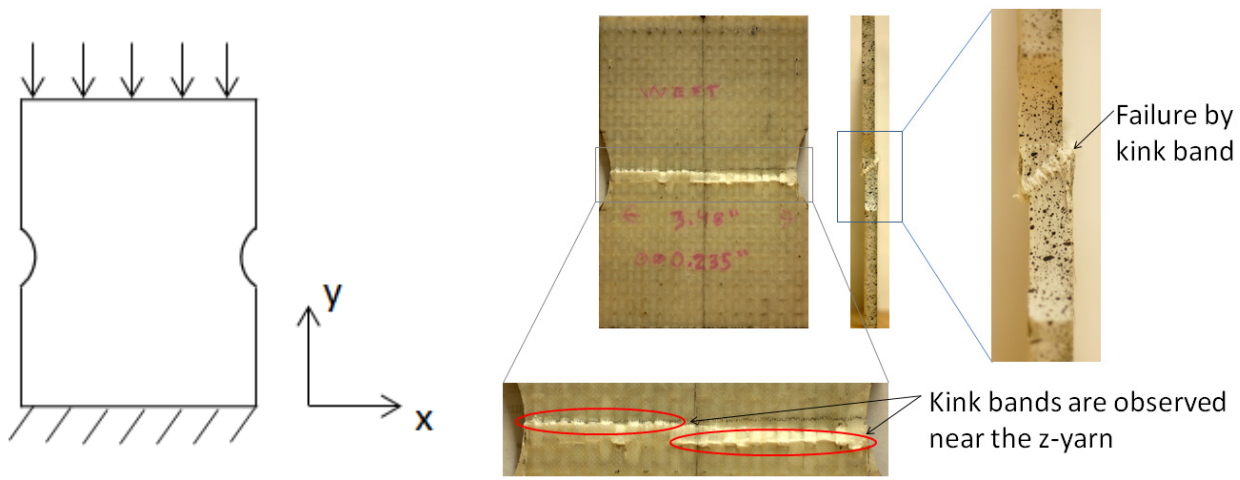

Figure 9. Configuration of the test setup and a typical failed specimen. 


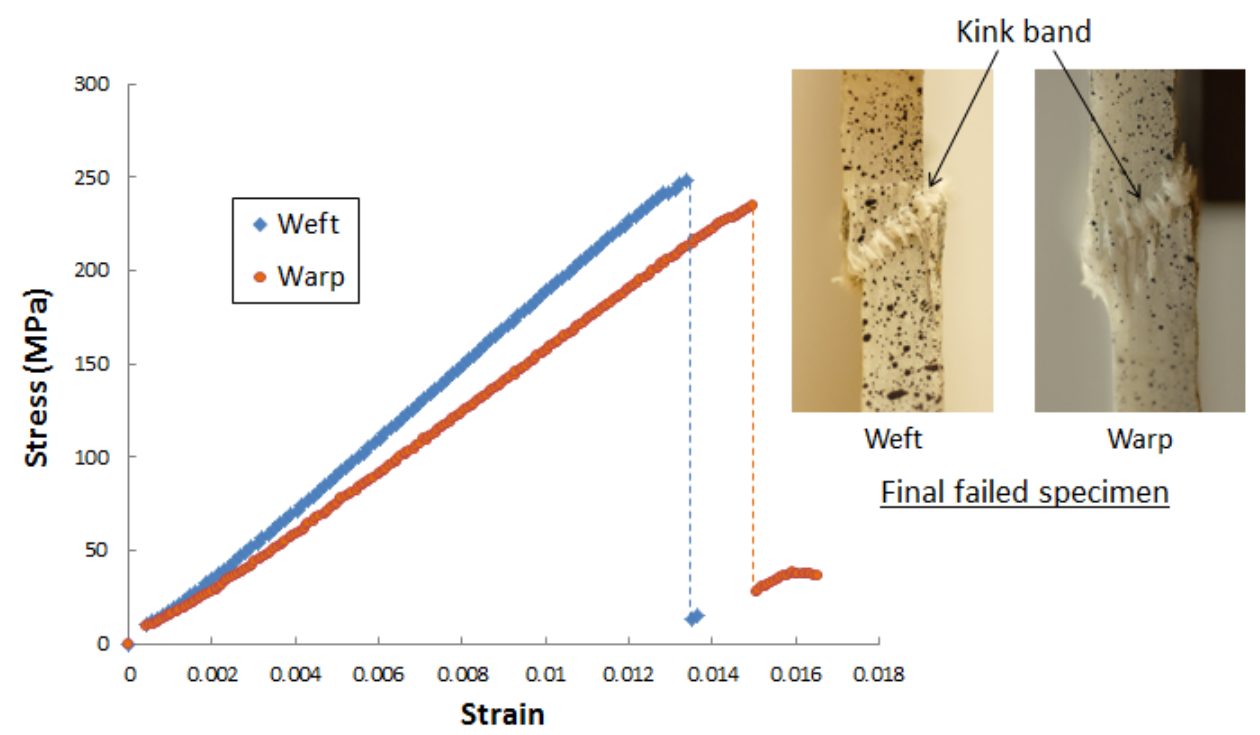

Figure 10. Compressive response of the 3DWTC under compression in the weft and warp directions.

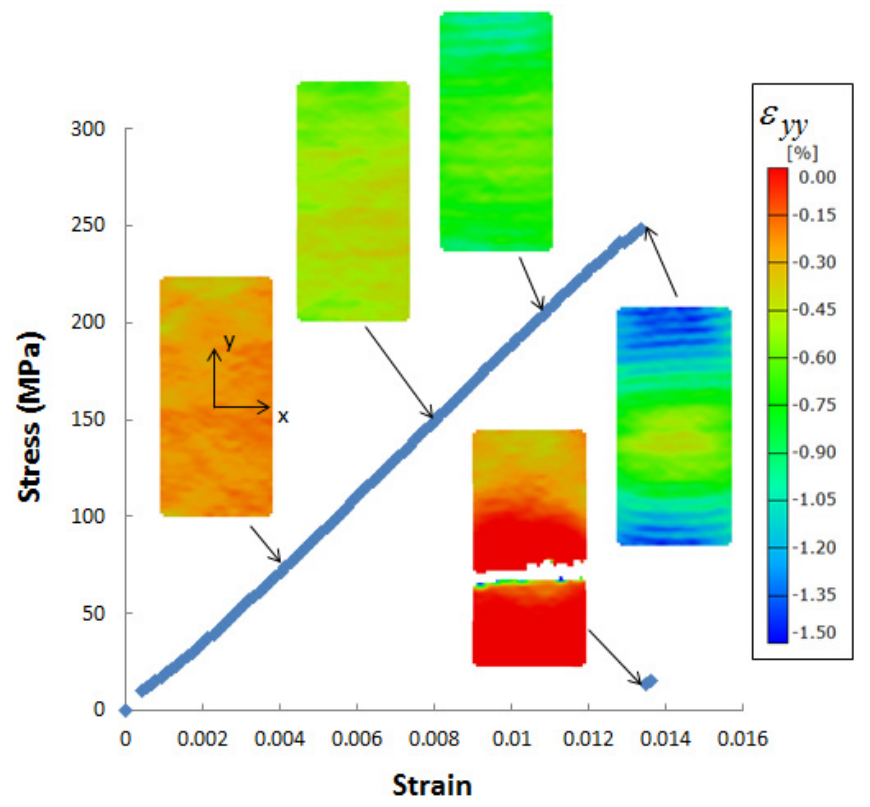

Figure 11. DIC strain field of the specimen loaded in the weft tow direction.

\section{B. Finite element analysis using RUC models}

The finite element based RUC model is utilized to study the compressive response of the 3DWTC. The RUC model represents the tows as elastic-plastic transversely isotropic solids as discussed earlier and the matrix as an elastic-plastic isotropic solid that conforms to the $J 2$ incremental theory of plasticity based on the nonlinear in-situ properties in Figure 4. The RUC that is computationally analyzed is shown in Figure 12. Since the responses of the 3DWTC loaded in the two different directions show similar behavior until it reaches the peak stress and fail by kink banding (see Figure 10), the compressive response of the textile composite compressed in the weft tow direction will be discussed here.

The RUC model is subjected to displacement control compressive loading, incorporating nonlinear geometry and material properties in conjunction with the arc-length, RIKS solver available in the commercial software ABAQUS/Standard. The RUC mesh is perturbed by the first egienmode that is characterized by a misalignment angle $\theta$. The comparative results in Figure 13 show that the RUC models produce the correct linear elastic stiffness 
of the 3DWTC. The peak stress from FEA decreases as the misalignment angle increases, implying that further increase of the initial imperfection magnitude may lead to good agreement between the prediction and experimental data. However, the FE model currently assumes perfect bonding at interfaces between the tow and matrix. Interfacial failure between fiber tows and matrix is prone to occur as the textile composite is loaded. Implementation of a model for the interface failure is expected to produce a more realistic prediction in addition to the initial imperfect geometry.

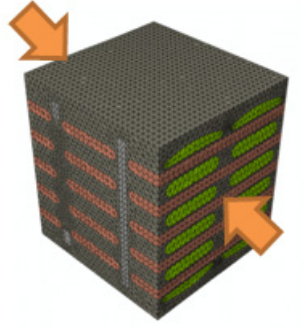

$\underline{\text { Initial configuration }}$

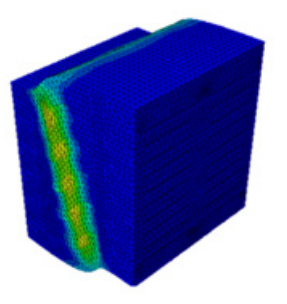

Final deformed configuration
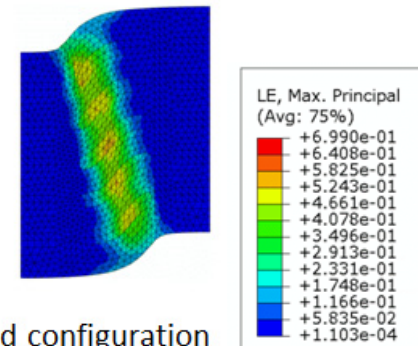

Figure 12. Finite element based RUC model in the initial and final deformed configurations. The kink band formation is captured from finite element analysis using the RUC model.

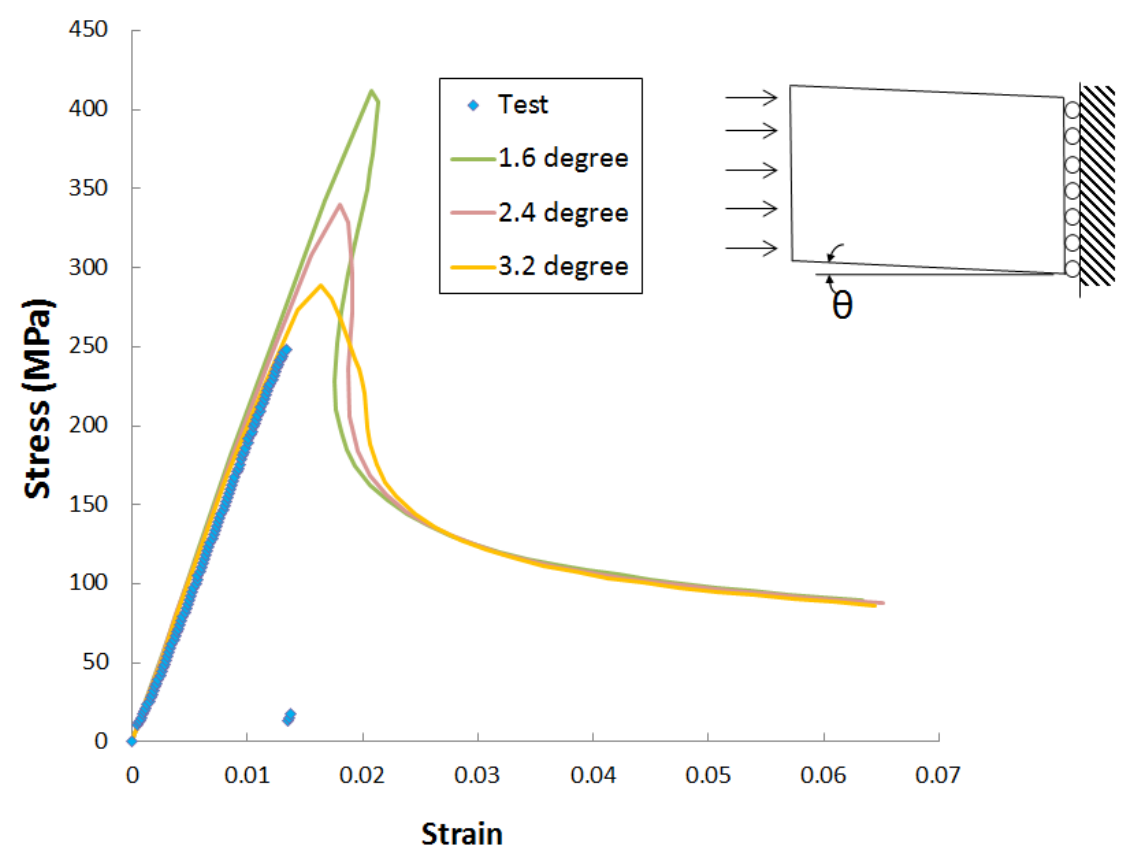

Figure 13. Comparison of the FEA results against the experimental data for the specimens loaded in the weft tow direction.

\section{Probabilistic Finite Element Analysis}

The present modeling and analysis methodology for textile composites also utilizes a statistically-based mechanics model embedded in a 3D nonlinear finite element (FE) code. Material and geometrical properties of composites in reality are not deterministic values because of unintended perturbations induced during manufacturing process. Other sources of uncertainties include loading and boundary conditions and initial damage configuration. The role of probabilistic modeling and analysis is to quantify the variations and uncertainties in predicted response of the hybrid composite materials due to such variations. When the uncertainty in the various parameters is considered through a probability-based analysis, more realistic comparison of model predictions and experimental results are possible for detailed model validation purposes. Additionally, key parameters driving numerous failure mechanisms can also be identified and the results can be utilized at a preliminary design stage to improve the model 
fidelity. In this presentation, NESSUS software coupled with ABAQUS is utilized to perform the probabilistic analysis.

NESSUS performs probabilistic analysis using the most probable point (MPP) methods. ${ }^{7}$ The MPP-based methods such as the first order reliability method (FORM), the second order reliability method (SORM) and the advanced mean value plus (AMV+) method do not gather all sampling data unlike conventional sampling methods such as a Monte-Carlo method. MPP methods calculate the probability of failure by first locating the MPP and then iterating numerical integration techniques to evaluate the probability. The key to efficiency in using MPP-based methods is locating the MPP as quickly as possible. In this section, the probabilistic progressive failure analysis is performed accounting for geometric variability as listed in Table 1, Table 2 and Table 3.

Figure 14 schematically illustrates the procedure of the probabilistic finite element analysis for the compressive response of the textile composite compressed in the weft tow direction. AMV+ method is used to obtain the reliability response of the textile composite.

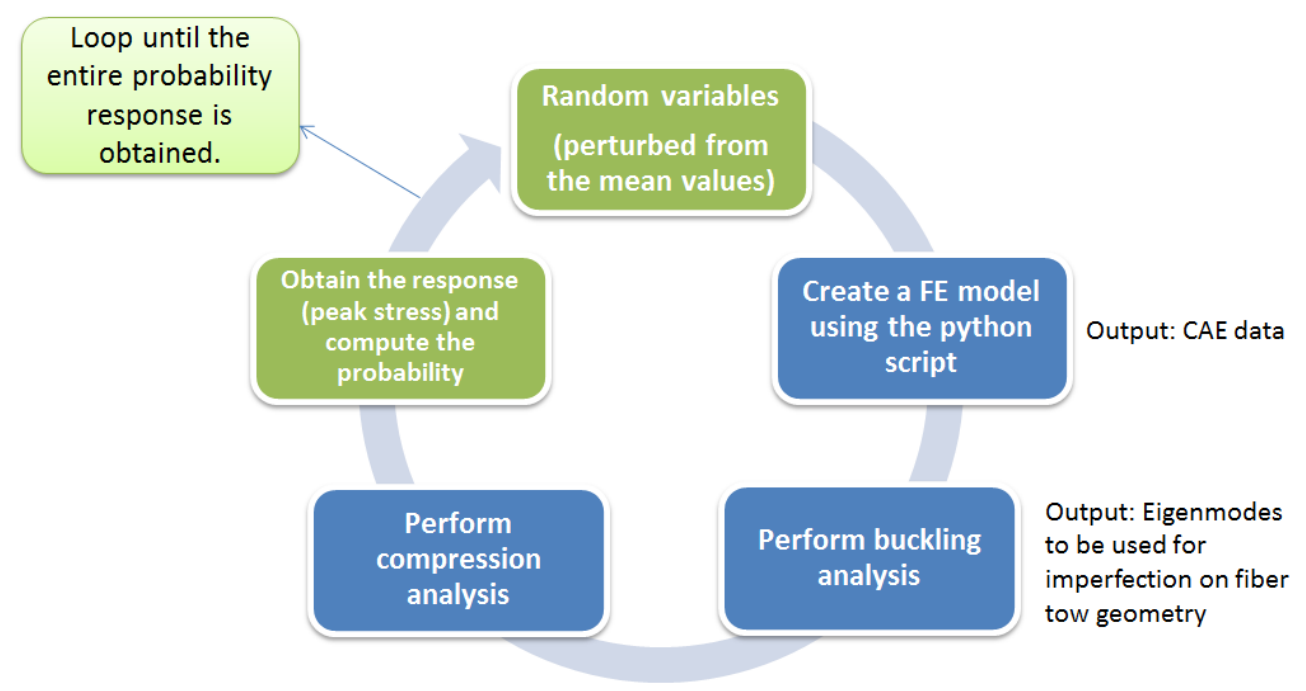

Figure 14. Procedure of the probability analysis using NESSUS software coupled with ABAQUS. The overall loop process is controlled by NESSUS.

Figure 15 shows the probability of peak stress for the RUC model when uncertainty in the geometrical modeling parameters is accounted for. The reliability response of the textile composite is obtained over a range as can be seen in Figure 15, indicating that the probabilistic modeling method can be used for detailed model validation purposes through the comparison of model predictions against experimental results. The magnitudes of the sensitivities indicate which parameters have an important influence on the probabilistic structural response. Random variables associated with weft tows and Z-yarns are listed in Figure 16 and Figure 17 since the modeling parameters of the warp tows have little influence on the response of the 3DWTC loaded in the weft tow direction. In Figure 16, the signs of the sensitivities indicate the direction that the mean value of the parameter should be changed in order to reduce the probability of failure or improve the compressive strength of the textile composite. Parameters with positive sensitivities should have their mean values reduced, while parameters with negative sensitivities should have their mean values increased. Figure 17 shows the importance level of each modeling parameter. The probabilistic importance factor, $\alpha$, is defined as a measure of the contribution of each parameter to the probability. ${ }^{7}$ The dimensions of Z-yarns are the most important factor to define the compressive response. It is interesting to note that the size of fiber tow number 1 is as important as Z-yarn dimensions as shown in Figure 16 and Figure 17 since the tow is not supported by Z-yarns and directly interacts with matrix regions as shown in Figure 2. 


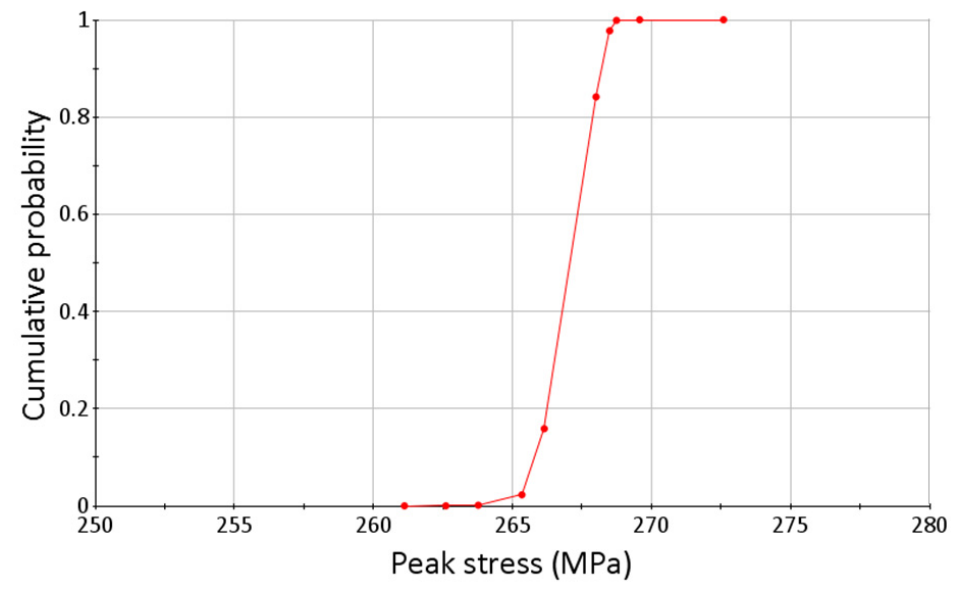

Figure 15. Cumulative probability distribution for peak stresses of the 3DWTC under compression.

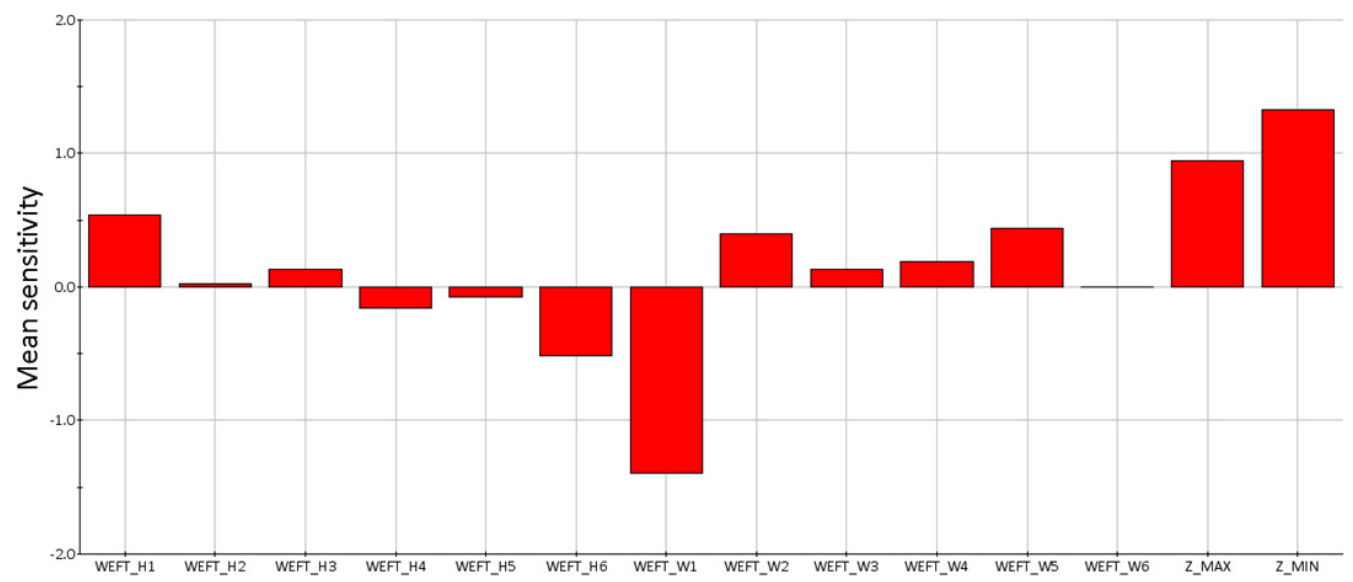

Figure 16. Sensitivities of the compressive response to mean values of modeling parameters.

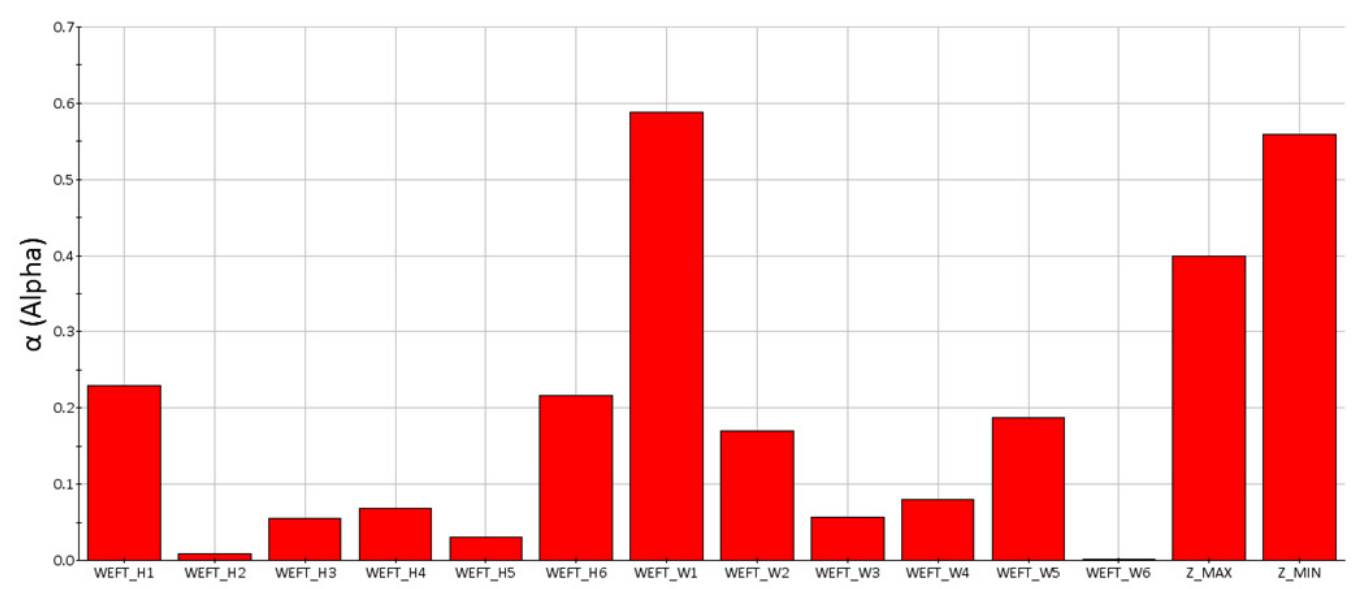

Figure 17. Importance levels of each modeling parameter. 


\section{Conclusion}

Quasi-static simulations of 3D woven textile composites have been reported. The modeling strategy, which involves homogenized elastic plastic tows within an elastic-plastic matrix, has been shown to capture the salient features of compression tests on the textile specimens. The computational model is able to predict compressive response and onset of failure under quasi-static compressive loading. The single RUC representation is utilized to predict both the failure mode and the peak stress accurately. Probabilistic finite element analysis is also performed to identify important modeling parameters for the overall structural performance of the textile composite with the complex fiber architecture. The probabilistic FEA has shown that it is capable of providing reliability-based failure indicators when variabilities in modeling parameters are considered.

\section{Acknowledgments}

The authors are grateful for the financial support from Boeing company.

\section{References}

${ }^{1}$ R. Kamiya, B.A. Cheeseman, P. Popper, and T.W. Chou. (2000). "Some recent advances in the fabrication and design of three-dimensional textile preforms: a review," Composites Science and Technology, 60, 33-47.

${ }^{2}$ M. Awais and U.N. Butt. 3D preforms in the world of composites-a review, National Textile University, Faisalabad, Pakistan.

${ }^{3}$ D. Stobbe and M. Mohamed. (2003). "3D woven composites: cost and performance viabiliity in comercial applications," In $48^{\text {th }}$ International SAMPE symposium.

${ }^{4}$ Song, S., Waas, A. M., Shahwan, K. W., Faruque, O., and Xiao, X. (2008). "Compression response of 2D braided textile composites: single cell and multiple cell micromechanics based strength predictions," Journal of Composite Materials, 42(23), 2461-82.

${ }^{5}$ Pankow, M., The Deformation Response of 3DWoven Composites Subjected to High Rates of Loading, PhD thesis, University of Michigan, 2010.

${ }^{6}$ Zhou, Y., Akanda, S. R., Jeelani, S. and Lacy, T. E. (2007). "Nonlinear constitutive equation for vapor-grown carbon nanofiber-reinforced SC-15 epoxy at different strain rate," Materials Science and Engineering: A, 465, 238-246.

${ }^{7}$ Southwest Research Institute, NESSUS Users Manual, Ver. 9.5, 2010. 